\section{Modell, generalisiert lineares}

\author{
R.-D. Hilgers ${ }^{1}$, N. Heussen ${ }^{1}$ und S. Stanzel ${ }^{2}$ \\ ${ }^{1}$ Institut für Medizinische Statistik, Universitätsklinikum der \\ RWTH Aachen, Aachen, Deutschland \\ ${ }^{2}$ Heidelberg, Deutschland
}

\section{Synonym(e) GLM}

Englischer Begriff generalized linear model

Definition Ein generalisiert lineares Modell ist ein verallgemeinertes Regressionsmodell, in dem die Zielvariable eine Verteilung ( $\triangleright$ Verteilung, statistische) aus der Klasse der Exponentialfamilie besitzt.

Beschreibung In linearen Modellen ( $\triangleright$ Modell, statistisches) nimmt man an, dass die Zielvariable normalverteilt ist, während sie in generalisiert linearen Modellen eine Verteilung aus der erweiterten Klasse der Exponentialfamilie besitzt. Diese Verteilungsklasse beinhaltet neben der $>$ Nor- malverteilung auch die Binomial-, Poisson-, Gamma- und inverse Normalverteilung.

Im generalisiert linearen Modell beeinflussen die Einflussfaktoren die Verteilung der Zielvariablen nicht direkt, sondern durch eine lineare Funktion, den so genannten linearen Prädiktor. Der erwartete Wert der Zielgröße in der \ Grundgesamtheit kann dann über die sog. Link-Funktion, eine Funktion des linearen Prädiktors, in Beziehung zu den Einflussfaktoren gesetzt werden. Die Art der verwendeten Link-Funktion hängt vom Typ der zugrunde liegenden Verteilung aus der Klasse der Exponentialfamilie ab. Einen häufig auftretenden Spezialfall eines generalisiert linearen Modells stellt die Logistische Regression ( $\triangleright$ Regression, logistische) dar. Die Logistische Regression modelliert Daten, bei denen die Zielgröße eine kategorielle Variable ist, deren Verteilung einer $>$ Binomialverteilung folgt. Demzufolge wird im logistischen Regressionsmodell der logit-link als Link-Funktion verwendet.

\section{Literatur}

McCullagh P, Nelder JA (1983) Generalized linear models. Chapman \& Hall, London 